\title{
The role side effects play in the choice of antiepileptic therapy in brain tumor-related epilepsy: a comparative study on traditional antiepileptic drugs versus oxcarbazepine
}

\author{
Marta Maschio*1, Loredana Dinapoli ${ }^{1}$, Antonello Vidiri², Andrea Pace ${ }^{3}$, \\ Alessandra Fabi ${ }^{4}$, Alfredo Pompili ${ }^{3}$, Maria Carmine Carapella ${ }^{3}$ and \\ Bruno Jandolo ${ }^{1}$
}

\begin{abstract}
Address: ${ }^{1}$ Center for tumor-related Epilepsy, Department of Neuroscience and Cervical- Facial Pathology, National Institute for Cancer "Regina Elena", Via Elio Chianesi 53, 00144 Roma, Italy, ${ }^{2}$ Department of Radiology, National Institute for Cancer "Regina Elena", Via Elio Chianesi 53, 00144 Roma, Italy, ${ }^{3}$ Department of Neuroscience and Cervical-Facial Pathology, National Institute for Cancer "Regina Elena", Via Elio Chianesi 53, 00144 Roma, Italy and "Division of Clinical Oncology, National Institute for Cancer "Regina Elena", Via Elio Chianesi 53, 00144 Roma, Italy

Email: Marta Maschio* - maschio@ifo.it; Loredana Dinapoli - loredana.dinapoli@gmail.com; Antonello Vidiri - vidiri@ifo.it; Andrea Pace - pace@ifo.it; Alessandra Fabi - fabi@ifo.it; Alfredo Pompili - pompili@ifo.it; Maria Carmine Carapella - carapella@ifo.it; Bruno Jandolo - jandolo@ifo.it
\end{abstract}

* Corresponding author

Published: 6 May 2009

Journal of Experimental \& Clinical Cancer Research 2009, 28:60 doi:10.1 I86/1756-9966-28-60

This article is available from: http://www.jeccr.com/content/28/I/60

(C) 2009 Marta et al; licensee BioMed Central Ltd.

This is an Open Access article distributed under the terms of the Creative Commons Attribution License (http://creativecommons.org/licenses/by/2.0), which permits unrestricted use, distribution, and reproduction in any medium, provided the original work is properly cited.
Received: 5 February 2009

Accepted: 6 May 2009

\begin{abstract}
Background: Seizure control doesn't represent the only challenging goal in patients with brain tumor-related epilepsy. Side effects have often taken precedence for patients' quality of life.

Methods: We performed an observational retrospective study on patients with brain tumorrelated epilepsy: 35 who had assumed oxcarbazepine monotherapy and 35 patients who had undergone treatment with traditional antiepileptic drugs. Primary variable of efficacy was the mean seizure frequency per month and safety variables were the drop-out for side effects and total incidence of side effects. We applied the Propensity Score technique to minimize selection bias.

Results: Our results showed a similar efficacy of oxcarbazepine and traditional antiepileptic drugs over time, but the difference in safety and tolerability between the two groups was significant: traditional AEDs caused more side effects, both serious and non serious.

Conclusion: This study highlights the importance of taking into consideration not only seizure control but also the appearance of side effects when choosing antiepileptic drugs in this patients population.
\end{abstract}

\section{Background}

Seizures are a common symptom in patients with brain tumors [1]. Literature data on antiepileptic drugs (AEDs) in brain tumor patients indicate that not only complete seizure control is a challenging goal [2] but that reducing unpleasant side effects produced by AEDs is a serious concern as well [3]. Side effects are mostly associated with the administration of traditional, older antiepileptic drugs: carbamazepine (CBZ), phenobarbital (PB), phenytoin (PHT) and valproic acid (VPA) [3-7]. Some limited data in 
the literature indicate that side effects are less marked when the newer AEDs such as oxcarbazepine, levetiracetam, topiramate, gabapentin and pregabalin are administered [6-13]. However, there have been no comparative studies to date which document the differences in efficacy and tolerability between the newer and older AEDs. The aim of this study was to assess if one of the newer generation AEDs presented significant differences in terms of efficacy as well as safety/tolerability when compared to the traditional AEDs, in patients with brain-tumor related epilepsy.

We chose not to undertake a comparative prospective study using traditional AEDs versus new AEDs, because substantial data indicate high toxicity of traditional AEDs and their interactions with chemotherapeutic agents strong enough to shorten life expectancy $[7,14-18]$. Therefore, we preferred to compare two retrospective groups, one in therapy with traditional AEDs and one with a new generation AED - oxcarbazepine - in order to assess if there were differences in efficacy and tolerability.

We choose a retrospective group of patients treated with oxcarbazepine because its efficacy is similar to that observed with the old AEDs [19], but, the low induction of CYP enzymes by OXC is associated with lower pharmacological interaction than other drugs. For this reason, also the interactions with chemotherapeutics agents appear unlikely $[20,21]$.

\section{Methods \\ Study design}

We made a retrospective chart review for 35 brain tumor patients who were followed in our Institute because of brain tumor and epilepsy during the period 1995 to December 2005 (the last date which had been documented in the medical charts). These patients had been in treatment with traditional AEDs (Traditional AEDs group). We chose those patients whose age, sex and duration of AED treatment were similar to the OXC group.

We conducted a retrospective chart review on 35 patients with brain tumor and epilepsy who came to our Center during the period January, 2002 to February, 2007 in order to evaluate the efficacy and tolerability of OXC monotherapy (OXC group). Data were collected from medical charts until June 2007 (data chosen for the end of the study).

We compared the Traditional AED group to the OXC group in order to assess if there were differences in efficacy and tolerability.

The study was approved by the Institute's Ethical Committee.

\section{Selection of patients}

Patients with brain tumor related epilepsy were included in the study if: between the ages 18 and 85; if they had had a KPS $\geq 60$; if they had received a diagnosis of their disease (primary brain tumors or metastatic brain tumors) after surgical intervention or radiological diagnosis. Patients were eligible for inclusion if they had experienced at least one observable seizure in the last year, prior to screening. Patients with epilepsy unrelated to brain tumor were excluded from the study. The following information was collected for each patient, at baseline and during the history of disease: surgery, type of chemotherapy, radiotherapy, presence of a tumoral progression.

\section{Assessment methods}

Traditional AED group and OXC group

A retrospective chart review was conducted on 35 brain tumor patients who had received PB, CBZ, PHT or VPA monotherapy for seizure control and on 35 brain tumor patients who had received OXC monotherapy for seizure control at our Center. These patients had arrived at our Center: 1) for uncontrolled seizures and/or side effects which had been caused by previous AED therapy 2) soon after the diagnosis of epilepsy related to brain tumor, without having had any prior AED therapy. Seizure frequency (SF) was assessed based on number of seizures documented in patient histories, hospital charts, and clinic notes. The appearance of side effects was assessed by using clinical notes and hospital charts. The severity of the AED's side effects was evaluated using the "Common Terminology Criteria for Adverse Events" [22].

\section{Statistical analyses}

The aim of the study was to conduct a comparative analysis between the treatment groups: A) OXC Group and B) Traditional AED Group in order to evaluate the efficacy in controlling seizures as well as the safety and tolerability of the AEDs. The primary efficacy variable which we used was the mean number of seizures per month. The safety variables used were both the drop-out for side effects as well as the total incidence of side effects.

In order to subject our data to statistical analyses, it was necessary to create homogeneity between the two treatment groups (OXC and Traditional AEDs). This was done by applying a Propensity Score (PS), even though this technique is primarily used for larger samples. A PS is used by identifying co-variables in both groups to insert in the logistic regression model. Seven co-variables useful for the analysis were identified: age, sex, tumor progression, KPS, chemotherapy, seizure frequency at base visit, follow-up duration. The statistical analysis of efficacy between treatment groups was applied using a General Linear Model for fixed factors (GLM), taking into consideration the following factors: 1) Treatment Group (OXC versus Tradi- 
tional AEDs) 2) Visit (baseline versus final follow-up) 3) Interaction between Treatment Group and Visit. The PS was applied only for the analysis of efficacy between treatment groups, and not for the safety/tolerability comparison between groups. For the analysis of safety variables (drop-out incidence and total incidence of side effects) we used the Fisher Exact Test taking into consideration the number of patients who had left the study or who had had side-effects.

The changes of SF from baseline to the final follow-up visit were evaluated using statistical analysis on the intentto-treat (ITT) population (that is patients who had had at least one on-treatment visit with seizure counts).

\section{Results \\ Traditional AED group \\ Patient Profiles}

Patients' demographic and clinical characteristic are depicted in table 1 [see additional file 1].

Sixteen (16) had had glioblastoma multiforme (GBM), 5 anaplastic astrocytoma (AA), 4 anaplastic oligodendroglioma (AO), 8 low grade astrocytoma (LGA) and 2 low grade oligodendroglioma (LGO). Fourteen patients had undergone only chemotherapy during the follow up, 7 patient had undergone only radiotherapy, 11 chemotherapy and radiotherapy and 3 patients had not undergone any systemic therapy. Eight patients had had tumoral progression during follow up.

The mean age at diagnosis of brain tumor was 50.1 years (range 22 to 76 years). Nine patient had had simple partial seizures (SP), 9 had had complex partial (CP), 3 had had SP + secondarily generalized tonic clonic seizures (SP+SGTC) and 14 had had CP+SGTC seizures. Patients had all been in monotherapy with traditional AEDs: $\mathrm{PB}$ $(\mathrm{N}=24) ; \operatorname{CBZ}(\mathrm{N}=9) ; \operatorname{VPA}(\mathrm{N}=1)$, PHT $(\mathrm{N}=1)$. Mean dosages: $\mathrm{PB}=112.5 \mathrm{mg} /$ day, $\mathrm{CBZ}=800 \mathrm{mg} /$ day, $\mathrm{VPA}$ $1000 \mathrm{mg} /$ day (only 1 patient), PHT $200 \mathrm{mg} /$ day (only 1 patient) [see additional file 1].

\section{Efficacy}

The mean seizure frequency per month before AED treatment had been 4.1 (35 patients) and 1.6 (35 patients) at final follow up. At final follow up, 45.7\% of patients (16 patients) were seizure free. GLM repeated measure analysis showed a significant reduction of seizure frequency at final follow-up ( $p=0.0095)$. Mean duration of follow up was 13.7 months (range 2 to 48 months).

\section{Adverse Events}

During treatment fifteen patients (42.9\%) had reported side effects: 11 patients in therapy with $\mathrm{PB}, 3$ with $\mathrm{CBZ}$ and 1 with VPA. Two patients (5.7\%), all in therapy with CBZ, had had mild and reversible side effects (haematological toxicity) and 13 patient (37.2\%) had had heavy side effects: 5 psychomotor slowness (4 patients with $\mathrm{PB}$ and 1 with VPA), 4 rash (all patient with PB), 2 periarthritis (all patients with $\mathrm{PB}$ ), 1 somnolence (patient with $\mathrm{PB}$ ) and 1 liver toxicity (patient with CBZ) [See additional file 2].

\section{OXC Group}

Patient Profiles

Patients' demographic and clinical characteristic are depicted in table 3 [see additional file 3].

Twelve patients had brain metastases, 4 GBM, 10 AA, 1 OA, 6 LGA and 2 meningioma. During follow up, 6 patients had undergone only chemotherapy, 3 patients had undergone only radiotherapy, 23 patients had undergone both chemotherapy and radiotherapy and 3 patients had not undergone any systemic therapy. Fourteen patients had had tumoral progression. The mean age at diagnosis of brain tumor was 52 years (range 18 to 81 years).

Eleven patients had had SP seizures, 4 had had CP, 6 had had SP+SGTC and 14 had had CP+SGTC seizures.

Eighteen patients had already been treated with other AEDs: $\mathrm{PB}=14 ; \mathrm{CBZ}=3$; topiramate $-\mathrm{TPM}-(\mathrm{N}=1)$ that had been changed to OXC for heavy side effects (8 patients), uncontrolled seizures ( 9 patients) and 1 for uncontrolled seizures and heavy side effects. Mean dosages had been: $\mathrm{PB}=103.6 \mathrm{mg} /$ day, $\mathrm{CBZ}=466.70 \mathrm{mg} /$ day, TPM 150 (only 1 patient). Seventeen had been naïve patients. During the period considered for the study, patients had all been in monotherapy with OXC with a mean daily dosage of $1162.5 \mathrm{mg}$ [See additional file 4].

\section{Efficacy}

The mean seizure frequency per month before OXC therapy had been 2.9, and at the final follow-up had been 0.6 (35 patients). Considering separately the two subgroups naive patients versus patients presenting for side effects/ inefficacy, the mean seizure frequency per month before OXC therapy had been 4.64 (naïve patients, 17 patients) and 1.3 (non-naïve patients, 18 patients). At the final follow-up the mean seizure frequency had been 0.88 (naïve patients) and 0.4 (non-naïve patients). At final follow up, we obtained $62.9 \%$ patients who were seizure free $(22$ patients). GLM repeated measure analysis showed a significant reduction of seizure frequency at final follow-up $(p=0.0018)$. Mean duration of follow up was 16.1 months (range 4 to 48 months). 


\section{Adverse Events}

During follow up 4 patients (11.4\%) reported side effects: 1 patient $(2.9 \%)$ had had mild and reversible side effects (mild rash and liver toxicity) and 3 (8.6\%) had had heavy side effects (2 rash and 1 cephalea) [See additional file 4].

\section{Comparison between the two groups Efficacy}

In order to compare monthly seizure frequency in both groups we used GLM repeated measure analysis with variables: treatment groups (Traditional AEDs versus OXC group), visit (baseline versus follow up), and interaction Group $\times$ Visit. Statistical analysis for both groups showed a significant reduction of seizure frequency between first visit and last follow up visit ( $\mathrm{p}<0.0001)$. The comparison made between treatment groups and interaction Group $\times$ Visit is not significant ( $\mathrm{p}=0.1166$ between groups; $\mathrm{p}=$ 0.9221 Group $\times$ Visit) .

\section{Adverse Events}

Taking into consideration the first variable of safety, drop out for side effects, the Fisher exact test showed a significant difference between the OXC group and the Traditional AED group $(p=0.0090)$ (Odds ratio $=6.303)$. In particular, concerning drop-out due to heavy side effects, only 3 patients in the OXC group and 13 patients of Traditional AEDs group were forced to stopped the AEDs.

Taking into consideration the second variable of safety, total incidence of side effects, Fisher exact test showed a significant difference between the OXC group and the Traditional AED group $(\mathrm{p}=0.0063)$ (Odds ratio $=5.813)$. In particular, four patients had side effects during OXC treatment whereas 15 patients in the Traditional AEDs group had side effects.

\section{Discussion}

Epilepsy is considered the most important risk factor for long-term disability in brain tumour patients [23]. Unfortunately, the side effects related to antiepileptic drugs can seriously affect the patients' quality of life; in fact, it has been found that patients' concerns with the AEDs' side effects have often taken precedence over their desire to reduce seizure frequency [24]. Side effects are mostly associated with the administration of traditional, older AEDs [3-8]. The few studies which have been done on the newer AEDs indicate that these same side effects are less frequent with these drug [9-13]. To date, a comparative study of this type has not been done.

We performed a statistical analysis and applied a Propensity Score in order to minimize the selection bias and other sources of bias. Concerning efficacy, results showed no major differences between the two groups. Concerning safety and tolerability, however, the profiles differ signifi- cantly. The traditional AED group had had more side effects than the OXC group (42.9\% vs $11.4 \%$ ), including heavy side effects which led patients to discontinue usage of the AED. It is generally accepted that the percentage of patients withdrawing because of adverse effects represents a reliable marker of tolerability [25].

The percentage of side effects for OXC was similar to that observed in non-tumoral, epileptic patients (10\%)[19], and the percentage of side effects for traditional AEDs is consistent with literature data (5 to $38 \%$ in patients with brain tumor-related epilepsy)[3].

The most common side effects we found were rash (11.4\% in Traditional AEDs group and $8.6 \%$ in OXC group) and psychomotor slowness (21.7\% only in Traditional AEDs group). In epileptic, non-tumoral patients, rash is a common side effect associated with most AED use, ranging between $3-10 \%$ and has been the leading cause of withdrawal from some AED trials [6,26]. The available data to date indicate that in patients with brain tumor-related epilepsy, the incidence of severe rash is higher than in non-tumoral, epileptic patients (14\%)[3]. This is in part due to the fact that patients with brain tumor often undergo radiotherapy and chemotherapy and this association induces an increased incidence of skin reactions in patients assuming old AEDs like PHT, $\mathrm{CBZ}$ and $\mathrm{PB}$ [4]. Our results are consistent with these literature data. Regarding psychomotor slowness our results are consistent with literature data that shows that patients with brain tumor-related epilepsy taking CBZ, VPA, PB and PHT performed worse in all cognitive domains than patients who did not undergo any AED therapy [6]. It is important to note that literature data cites cognitive impairment in brain tumor patients as much more common than the physical disability $[27,28]$. Such impairment is the major variable which influences quality of life in patients with epilepsy [29]. For this reason, the choice of an AED which does not impair cognitive functioning is of primary importance for patients with brain tumorrelated epilepsy.

Concerning efficacy, we observed a similarly good profile of efficacy over time in the two groups of treatment, with a significant reduction in number of seizures. However, the comparison between treatment groups is not significant. Studies to date dedicated specifically to the efficacy of the new AEDs in controlling seizures in patients with brain tumor-related epilepsy, are very recent [9-12].

In the literature only one study examined OXC monotherapy only in patients with brain tumor-related epilepsy [11]. This study was conducted for preventing perioperative seizures in patients with brain tumors. In the other studies, OXC is one of many drugs tested $[14,15,30]$. 
Recently, one study was done using OXC monotherapy in patients with cryptogenetic or symptomatic epilepsy [31]. In this study the efficacy of OXC is significantly more pronounced in patients with cryptogenetic epilepsy than in patients with brain tumors. Our study is the first that uses only OXC in epilepsy related to brain tumor, with a longterm follow up and with a good efficacy.

With regard to follow-up, it is important to point out the difficulty that the death of patients poses in studies of patients with this type of cancer. It should be noted that the mortality rate of patients with brain tumors makes long-term studies difficult and presents problems already at the onset with obtaining a significant number of participants for studies. In the two groups, the follow up varied from 2 to 48 months: this variability is due to deceased patients. This has already been mentioned as being a serious drawback to studies on this patient population.

In our study, both groups of patients were in treatment with chemotherapy, and data in the literature indicate that chemotherapy could play a role in seizure control [32]. Therefore, the fact that systemic therapy might have affected the outcome cannot be excluded.

In our study we have patients with different histological diagnosis, so we were unable to determine a difference in efficacy of AED therapy (traditional and OXC) in controlling seizures, based on the different histological diagnosis.

While we used the Propensity Score Technique to avoid selection bias, we cannot exclude the fact that data obtained in retrospective studies may affect the outcome concerning significant statistical differences in efficacy between the two groups.

\section{Conclusion}

This is the first study which compares the older AEDs with a newer AED, in patients with brain tumor-related epilepsy. Our most significant findings concern the presence of side effects, both serious and less serious in patients who had assumed the older AEDs. It was the serious side effects which were largely present in the traditional AEDs group; the extent to which patients with these side effects were forced to interrupt treatment. This brings us to the issue of patients' quality of life, which we urge must take into consideration not only seizure control, but also adverse events; most studies to date focus primarily on the former and not the latter. Our study clearly demonstrates that while both traditional AEDs and oxcarbazepine may reduce seizure frequency equally as well, the higher incidence of serious side effects which make the traditional AEDs less tolerable, affect the quality of life of patients who must already face numerous drug therapies.

\section{Competing interests}

The authors declare that they have no competing interests.

\section{Authors' contributions}

MM conceived and conducted the study and wrote the paper. LD participated in study design and contributed to paper writing. JB participated in study coordination. VA performed patients radiological examination. PA, FA, PA and $\mathrm{CMC}$ collaborate to data acquisition. All authors read and approved the final manuscript.

\section{Additional material}

\section{Additional file 1}

TRADITIONAL AEDs GROUP: Patients' clinical and vital data. The data in table provide clinical and vital data of patients of traditional AEDs group.

Click here for file

[http://www.biomedcentral.com/content/supplementary/1756-

9966-28-60-S1.doc]

\section{Additional file 2}

TRADITIONAL AEDs GROUP: Epilepsy characteristics. The data in table provide epilepsy characteristics of patients of traditional AEDs group. Click here for file

[http://www.biomedcentral.com/content/supplementary/17569966-28-60-S2.doc]

\section{Additional file 3}

OXC GROUP: Patients' clinical and vital data. The data in table provide clinical and vital data of patients of OXC group.

Click here for file

[http://www.biomedcentral.com/content/supplementary/17569966-28-60-S3.doc]

\section{Additional file 4}

OXC GROUP: Epilepsy characteristics. The data in table provide epilepsy characteristics of patients of OXC group.

Click here for file

[http://www.biomedcentral.com/content/supplementary/17569966-28-60-S4.doc]

\section{Acknowledgements}

The Authors wish to express their gratitude to Mrs Lesley Pritikin for reviewing the manuscript.

The Authors also thank Dr. Mauro Montanari for performing statistical analysis.

\section{References}

I. Vecht CJ, van Breemen M: Optimizing therapy of seizures in patients with brain tumors. Neurology 2006, 67(12 Suppl 4):SIO-SI3.

2. Hildebrand J, Lecaille C, Perennes J, Delattre JY: Epileptic seizures during follow-up of patients treated for primary brain tumors. Neurology 2005, 65:2 I2-215.

3. Glantz MJ, Cole BF, Forsyth PA, Recht LD, Wen PY, Chamberlain MC, Grossman SA, Cairncross JG: Practice parameter: anticonvul- 
sant prophylaxis in patients with newly diagnosed brain tumors. Neurology 2000, 54:1886-1893.

4. Aguiar D, Pazo R, Durán I, Terrasa J, Arrivi A, Manzano H, Martín J, Rifá J: Toxic epidermal necrolysis in patients receiving anticonvulsants and cranial irradiation: a risk to consider. J Neurooncol 2004, 66:345-350.

5. Arif $\mathrm{H}$, Buchsbaum R, Weintraub D, Koyfman S, Salas-Humara C, Bazil CW, Resor SR Jr, Hirsch LJ: Comparison and predictors of rash associated with I5 antiepileptic drugs. Neurology 2007, 68:1701-1709.

6. Klein M, Engelberts NH, Ploeg HM van der, Kasteleijn-Nolst Trenité DG, Aaronson NK, Taphoorn MJ, Baaijen H, Vandertop WP, Muller M, Postma TJ, Heimans JJ: Epilepsy in low-grade gliomas: the impact on cognitive function and quality of life. Ann Neurol 2003, 54:5I4-520.

7. van Breemen MS, Wilms EB, Vecht CJ: Epilepsy in patients with brain tumours: epidemiology, mechanisms, and management. Lancet Neurol 2007, 6:42I-430.

8. French JA, Kugler AR, Robbins JL, Knapp LE, Garofalo EA: Doseresponse trial of pregabalin adjunctive therapy in patients with partial seizures. Neurology 2003, 60:1631-1637.

9. Maschio M, Albani F, Baruzzi A, Zarabla A, Dinapoli L, Pace A, Pompili A, Carapella CM, Occhipinti E, Jandolo B: Levetiracetam therapy in patients with brain tumour and epilepsy. J Neurooncol 2006, 80:97-100.

10. Maschio M, Dinapoli L, Zarabla A, Pompili A, Carapella CM, Pace A, Giannarelli D, Occhipinti E, Jandolo B: Outcome and tolerability of topiramate in brain tumor associated epilepsy. J Neurooncol 2008, 86:6I-70.

II. Mauro AM, Bomprezzi C, Morresi S, Provinciali L, Formica F, lacoangeli M, Scerrati M: Prevention of early postoperative seizures in patients with primary brain tumors: preliminary experience with oxcarbazepine. J Neurooncol 2007, 8 I:279-285.

12. Newton HB, Goldlust SA, Pearl D: Retrospective analysis of the efficacy and tolerability of levetiracetam in brain tumor patients. J Neurooncol 2006, 78:99-102.

13. Perry JR, Sawka C: Add-on gabapentin for refractory seizures in patients with brain tumours. Can J Neurol Sci 1996, 23:|28-|3|.

14. Cloughesy TF, Wen PY, Robins HI, Chang SM, Groves MD, Fink KL, Junck L, Schiff D, Abrey L, Gilbert MR, Lieberman F, Kuhn J, DeAngelis LM, Mehta M, Raizer J], Yung WK, Aldape K, Wright J, Lamborn KR, Prados MD: Phase II trial of tipifarnib in patients with recurrent malignant glioma either receiving or not receiving enzyme-inducing antiepileptic drugs: a North American Brain Tumor Consortium Study. J Clin Oncol 2006, 24:365I-3656.

15. Kuhn JG: Influence of anticonvulsants on the metabolism and elimination of irinotecan. A North American Brain Tumor Consortium preliminary report. Oncology 2002, 16:33-40.

16. Oberndorfer S, Piribauer M, Marosi C, Lahrmann H, Hitzenberger P, Grisold W: P450 enzyme inducing and non-enzyme inducing antiepileptics in glioblastoma patients treated with standard chemotherapy. J Neurooncol 2005, 72:255-260.

17. Crews KR, Stewart CF, Jones-Wallace D, Thompson SJ, Houghton PJ, Heideman RL, Fouladi M, Bowers DC, Chintagumpala MM, Gajiar A: Altered irinotecan pharmacokinetics in pediatric high-grade glioma patients receiving enzyme-inducing anticonvulsant therapy. Clin Cancer Res 2002, 8:2202-2209.

18. Grossman SA, Hochberg F, Fisher J, Chen TL, Kim L, Gregory R, Grochow LB, Piantadosi S: Increased 9-aminocamptothecin dose requirements in patients on anticonvulsants. NABTT CNS Consortium. The New Approaches to Brain Tumor Therapy. Cancer Chemother Pharmacol 1998, 42:1 18-126.

19. Martinez W, Ingenito A, Blakeslee M, Barkley GL, McCague K, D'Souza J: Efficacy, safety, and tolerability of oxcarbazepine monotherapy. Epilepsy Behav 2006, 9:448-456.

20. Baruzzi A, Albani F, Riva R: Oxcarbazepine: pharmacokinetic interactions and their clinical relevance. Epilepsia 1994, 35(suppl 3): 14-19.

2I. Larkin JG, McKee PJ, Forrest G, Beastall GH, Park BK, Lowrie JI, Lloyd $P$, Brodie MJ: Lack of enzyme induction with oxcarbazepine (600 mg daily) in healthy subjects. Br J Clin Pharmacol I99I, 3I:65-7I.

22. Cancer Therapy Evaluation Program, Common Terminology Criteria for Adverse Events v3.0, DCTD, NCl, NIH,
DHHS, December I2, 2003 [http://ctep.cancer.gov/protocolDe velopment/electronic applications/docs/ ctcae index.pdf\#search="ctcae"]

23. Taillibert S, Laigle-Donadey F, Sanson M: Palliative care in patients with primary brain tumors. Curr Opin Oncol 2004, 16:587-592.

24. Hildebrand J: Management of epileptic seizures. Curr Opin Oncol 2004, 16:3|4-317.

25. Zaccara G, Messori A, Cincotta M, Burchini G: Comparison of the efficacy and tolerability of new antiepileptic drugs: what can we learn from long-term studies? Acta Neurol Scand 2006, I I 4:157-168.

26. Alvestad S, Lydersen S, Brodtkorb E: Rash from antiepileptic drugs: influence by gender, age, and learning disability. Epilepsia 2007, 48: I360-1365.

27. Meyers CA: Neuropsychological deficits in brain tumor patients: Effect of location, chronicity, and treatment. Cancer Bull 1986, 38:30-32

28. Meyers CA, Boake C: Neurobehavioral disorders in brain tumor patients: Rehabilitation strategies. Cancer Bull 1993, 45:362-364.

29. Brunbech L, Sabers A: Effect of antiepileptic drugs on cognitive function in individuals with epilepsy: a comparative review of newer versus older agents. Drugs 2002, 62:593-604.

30. Villikka K, Kivistö KT, Mäenpää H, Joensuu H, Neuvonen PJ: Cytochrome P450-inducing antiepileptics increase the clearance of vincristine in patients with brain tumors. Clin Pharmacol Ther 1999, 66:589-593.

31. Dogan EA, Usta BE, Bilgen R, Senol Y, Aktekin B: Efficacy, tolerability, and side effects of oxcarbazepine monotherapy: A prospective study in adult and elderly patients with newly diagnosed partial epilepsy. Epilepsy Behav 2008, I3:I56-16I.

32. Brada M, Viviers L, Abson C, Hines F, Britton J, Ashley S, Sardell S, Traish D, Gonsalves A, Wilkins P, Westbury C: Phase II of primary temozolomide chemotherapy in patients with WHO grade II gliomas. Ann Oncol 2003, 14:1715-172I.
Publish with BioMed Central and every scientist can read your work free of charge

"BioMed Central will be the most significant development for disseminating the results of biomedical research in our lifetime."

Sir Paul Nurse, Cancer Research UK

Your research papers will be:

- available free of charge to the entire biomedical community

- peer reviewed and published immediately upon acceptance

- cited in PubMed and archived on PubMed Central

- yours - you keep the copyright
BioMedcentral 